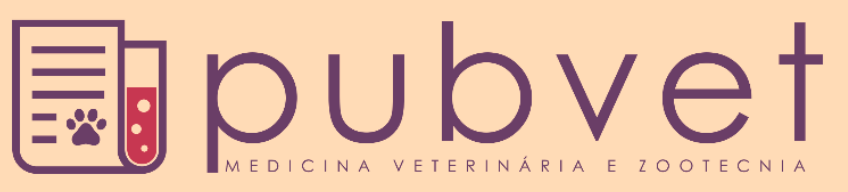

https://doi.org/10.31533/pubvet.v15n09a910.1-8

\title{
Uso de animais na experimentação animal: Revisão
}

\author{
Eldo Jose Rodrigues dos Santos ${ }^{1 *} \bullet \mathbb{D}$, Liara da Silva Assis ${ }^{2}{ }^{\circ}$, Marluce Pereira Damasceno \\ Lima $^{3}{ }^{\circ}$, Maria Christina Sanches Muratori ${ }^{4} \odot$ (D), Natanael Pereira da Silva $\operatorname{Santos}^{5}{ }^{\circ}$ (D) \\ ${ }^{I}$ Médico Veterinário, Doutorando do Programa de Pós-Graduação em Ciêcia Animal da Universidade Federal do Piauí, Teresina (PI) Brasil. \\ ${ }^{2}$ Mestranda do Programa de Pós-Graduação em Zootecnia Tropical/UFPI. \\ ${ }^{3}$ Bióloga, Professora, Departamento de Ensino, Instituto Federal de Educação, Ciência e Tecnologia do Maranhão, Campus São João dos Patos, Maranhão, Brasil. \\ ${ }^{4}$ Veterinária, Professora Doutora, Departamento de Morfofisiologia Veterinária, Universidade Federal do Piauí, Teresina (PI) Brasil. \\ ${ }^{5}$ Professor, Departamento: Coordenação do Curso de Engenharia Agronômica/UFPI/CPCE. \\ *Autor para correspondência, E-mail: eldojose10@hotmail.com
}

Resumo. O presente trabalho tem como objetivo revisar os aspectos referentes à experimentação animal apresentando aspectos históricos, éticos e práticos intrínsecos ao tema. Desde a Antiguidade, animais são utilizados em pesquisas científicas que testam a eficácia, mecanismos de ação e toxicologia de substâncias químicas com potencial para utilização como novos tratamentos para doenças. A utilização de animais em experimentação vem despertando uma forte discussão dentro da comunidade acadêmica e dos grupos de proteção dos animais. Buscando reduzir o uso de animais em pesquisas científicas, métodos alternativos estão sendo empregados fundamentando-se nos princípios dos "3Rs" para a realização de experimentação animal, ou seja, com foco na substituição, redução e refinamento. Experimentos bem planejados, que utilizam um número adequado de animais, aliados a uma análise estatística adequada, garantem a robustez e validade dos resultados experimentais. Vale ressaltar que aspectos referentes aos tipos de pesquisa e modelos experimentais devem ser levados em consideração, respeitando sempre os princípios legais e éticos, o que reflete na validade e aceitabilidade dos resultados.

Palavras-chave: Bioética, métodos alternativos, pesquisa científica

\section{The use of animals in experiments: Review}

\begin{abstract}
The present work aims to review the aspects related to animal experimentation, presenting historical, ethical and practical aspects intrinsic to the theme. Since ancient times, animals have been used in scientific research that tests the effectiveness, mechanisms of action and toxicology of chemical substances with potential for use as new treatments for diseases. The use of animals in experiments has aroused a strong discussion within the academic community and animal protection groups. Seeking to reduce the use of animals in scientific research, alternative methods are being used based on the principles of the "3Rs" for carrying out animal experimentation, that is, with a focus on replacement, reduction and refinement. Well-designed experiments, which use an adequate number of animals, combined with an adequate statistical analysis, guarantee the robustness and validity of the experimental results. It is worth mentioning that aspects related to the types of research and experimental models must be taken into consideration, always respecting the legal and ethical principles, which reflects in the validity and acceptability of the results.
\end{abstract}

Keywords: Bioethics, alternative methods, scientific research

\section{Introdução}

O uso de animais na experimentação pode ser entendido como uma técnica que proporciona a intervenção em animais, podendo estes estar vivos ou logo após abate com o intuito de cultivar o 
conhecimento científico. Embora essa prática venha sendo desenvolvida desde a antiguidade, esses procedimentos são capazes de atingir de uma certa forma a sensibilidade humana e, no cenário mais recente, vem despertando uma forte discussão dentro da comunidade acadêmica e dos grupos de proteção dos animais (Guimarães et al., 2016; Matthiessen et al., 2003; Schatzmayr \& Müller, 2008). Com esse panorama, indivíduos ligados aos grupos de defesa e proteção de animais enfatizam pela dispensa dessa prática, pelo fato de que se pode utilizar outros meios e métodos alternativos, ainda pela possibilidade de ocorrer erros metodológicos na qual, se pretende obter resultados e interpretações feitos com testes em uma determinada espécie de animal generalizando para uso dos resultados em humanos (Morales, 2008).

Quando se busca relatos do uso de animais pelo homem, encontra-se uma relação antiga. Os animais têm sido usados na alimentação, na caça, nos esportes, no transporte, nas cerimônias religiosas e sacríficos, tudo isso buscando um maior conforto de uma espécie, a humana. $\mathrm{Na}$ experimentação os animais vêm sendo utilizados há muitos anos, como forma de se compreender a anatomia, fisiologia e reações que se possa aprimorar o conhecimento nos mecanismos das doenças (Bones \& Molento, 2012; Franco et al., 2014; Magalhães, 2012).

Trabalhos como, Corpus Hippocraticum (cerca de 350 a.C.), possui relatos do uso de animais em experimentos utilizando suínos. No entanto, somente em meados do século XIX, foi que a experimentação com animais teve novos avanços no campo da medicina em seus estudos voltados para anatomia e fisiopatologia, para um melhor entendimento do corpo e reações (Franco et al., 2014). No ano de 1822, surgiu uma Lei Inglesa combatendo a crueldade no uso de animais (British Anticruelty $A c t$ ) com o intuito eliminar e controlar os movimentos de tortura, que se aplicava apenas para animais de grande porte domesticados (Goldim \& Raymundo, 1997). Logo no início do século XX foi proposto pela associação médica americana estudos sobre os aspectos éticos na utilização de animais no campo da experimentação animal (Guimarães et al., 2016).

No Brasil a primeira legislação de proteção dos animais que proibia a prática de maus tratos e crueldade surgiu no ano de 1934, criada pelo presidente Getúlio sob o Decreto 24.645/34. Com isso os animais passaram a serem protegidos por lei, que estabelecia multa e prisão para quem descumprisse o Decreto, sendo atribuídos a eles, também representação do Ministério Público e dos órgãos de proteção de defesa dos animais (Guimarães et al., 2016). Desde então as leis e decretos vem passando por atualizações no advento de melhorias nos modelos experimentais que utilizam animais em suas pesquisas.

Diante disso, a presente revisão tem por objetivo abordar sobre os aspectos históricos e normas atuais que regulamentam o uso de modelos experimentais com animais e todo o seu trâmite de aceitação no comitê de ética no Brasil.

\section{Histórico da experimentação em animais}

Em muitos cursos de graduação e pós-graduação relacionados às Ciências Biológicas e da Saúde, o processo de formação dos estudantes envolve a experimentação animal como prática inerente às atividades científicas de ensino e pesquisa (Tréz \& Rosa, 2013).

Animais de diversas espécies são utilizados em pesquisas científicas que testam a eficácia, mecanismos de ação e toxicologia de substâncias químicas com potencial para utilização como novos tratamentos para doenças (Doke \& Dhawale, 2013).

Em razão do reflexo social, ou seja, devido à possibilidade de fornecer benefícios para a saúde coletiva, estudos científicos com modelos animais não foram significativamente questionados durante grande parte da história. Entretanto, a partir do final do século XX surge o anseio pela implantação e validação de modelos alternativos aos experimentos executados tradicionalmente. Frente à essa demanda, vem sendo criada e regulamentada legislação nova e específica a respeito dessa pauta (Disner, 2019). Segundo Medeiros \& Albuquerque (2015), atualmente, há um verdadeiro desacordo entre as práticas vigentes nas universidades, as regulamentações emanadas da legislação e o que dispõe o texto constitucional. Por um lado, universidades enfatizam a necessidade do uso de animais em testes, ensino e pesquisa uma vez que essas práticas são fundamentais para o desenvolvimento científico e, consequentemente, para a saúde pública. Essa utilização, além de ser respaldada pela legislação, é 
atestada por comitês de ética e pela análise minuciosa dos protocolos utilizados durante o desenvolvimento da pesquisa nos trâmites de análise de dados para publicação sob a forma de artigos científicos (Medeiros \& Albuquerque, 2015).

Algumas ações judiciais foram movidas anos por entidades de defesa animal contra diversas universidades brasileiras gerando grande embate jurídico e político. A exemplo disso, pode-se citar a ação civil pública ambiental contra a Universidade Estadual de Maringá em outubro de 2011, a partir de uma denúncia e um abaixo assinado com mais de seis mil assinaturas reportando a possível prática de maus tratos a cães da raça beagle utilizados em experimentos científicos realizados na referida instituição (Medeiros \& Albuquerque, 2015).

Ainda no Brasil, em abril de 2013, uma entidade da sociedade civil denominada Instituto Abolicionista Animal, propôs ação civil pública questionando a Universidade Federal de Santa Catarina sobre o uso de animais no ensino em descumprimento da legislação pertinente.

A partir de 2014, os testes de cosméticos ou de seus ingredientes em animais foram banidos em alguns estados brasileiros, como São Paulo, Mato Grosso do Sul (2014), Pará, Paraná e Amazonas (2016), Rio de Janeiro (2017) e Minas Gerais (Lei n 23.050, de 28 de agosto de 2018) (Disner, 2019).

Segundo Tréz \& Rosa (2013) há nas universidades um perfil potencialmente tradicional de professores e estudantes no que se refere à utilização de animais em experimentação, com consolidada abordagem da educação científica afinada ao estilo de pensamento hegemônico vivisseccionistahumanitário.

Percebe-se hoje a iminente necessidade de disponibilidade e visibilidade dos testes alternativos com vistas a promover e facilitar a transferência de tecnologia. Essa nova realidade revela o desafio de adequar as pesquisas científicas às premissas de bem-estar animal, exigindo dedicação especial tanto na execução dos experimentos como na análise dos resultados oriundos de métodos alternativos de pesquisa (Disner, 2019).

\section{Animais utilizados em experimentação científica}

Apesar do recente interesse da legislação federal em pesquisa experimental com a utilização de animais, há um decréscimo do apoio financeiro para pesquisa. Por isso, é essencial a escolha justificável e econômica de animais experimentais. Nas fases pré-clínicas de descoberta e desenvolvimento de drogas os modelos animais constituem ferramenta indispensável para a testagem de novas terapias. $\mathrm{O}$ planejamento experimental deve considerar as características das espécies animais selecionados, um plano de avaliação apropriado com métodos validados, sincronização do estudo com requisitos regulatórios, tamanhos de amostra estatisticamente pré-justificados e métodos de avaliação (Peric et al., 2015).

No desenvolvimento de vacinas, estudos em animais podem fornecer evidências substanciais de eficácia para aprovação da vacina, desde que utilizada uma abordagem com a seleção cuidadosa de marcadores imunológicos apropriados, ou seja, protetores correlatos que estão estatisticamente relacionados a um desfecho clínico, que prediga o provável desfecho clínico (Yellowlees \& Perry, 2015).

Alguns trabalhos analisam a possibilidade de utilização de animais da fauna brasileira para fins de experimentação (Garcia Filho et al., 2013). Isola et al. (2013) descreveram a morfologia, morfometria e a ultraestrutura da pele do segundo maior roedor da fauna brasileira, Cuniculus paca, em razão de apresentarem aspectos característicos tais como, tamanho adequado, preço acessível e curto período de prenhez, indicadores confiáveis do habitat e modo de vida desses animais. Esses autores identificaram arquitetura da cútis e anexos cutâneos de $C$. paca semelhantes aos dos mamíferos em geral, embora haja ausência de glândulas sudoríparas e com diferenças entre os sexos.

\section{Princípio dos 3R's e métodos alternativos}

Elaborado pelo zoólogo Willian Russell e pelo microbiologista Rex Burch em 1959, o princípio dos 3R's refere-se à aplicação em experimentação animal dos pilares redução (reduction), substituição (replacement) e refinamento (refinement). Segundo esse princípio deve-se, a curto prazo, realizar a otimização do número de animais utilizados em experimentos (do ponto de vista quantitativo) e praticar a humanização dos procedimentos (do ponto de vista qualitativo), para sempre que possível e a longo 
prazo, alcançar a completa substituição do uso de animais em experimentos. Esse princípio fornece as premissas para o aperfeiçoamento de condutas experimentais em animais em consonância com a ética nos protocolos experimentais (Tréz, 2010). Na busca de reduzir o uso de animais em experimentação, métodos alternativos estão sendo empregados. A exemplo disso, alternativamente podem ser realizados testes in vivo nos quais estágios larvais ou microrganismos podem substituir animais adultos, bem como, células ou tecidos extraídos e cultivados externamente em meios de cultura sob condições laboratoriais otimizadas e controladas podem ser utilizadas para avaliar substâncias químicas ou produtos em estágios iniciais de desenvolvimento em testes in vitro (Smith et al., 2017). Ainda como alternativa, simulações e modelos computacionais (in silico) permitem a identificação de características e efeitos de compostos, sem a necessidade de utilização de material biológico (Balls et al., 2018).

As vantagens dos métodos alternativos incluem rapidez, menor necessidade de mão de obra, maior custo-benefício e, no caso de testes in vitro, controle das condições experimentais (Doke \& Dhawale, 2013). No Brasil, a validação de métodos alternativos ao uso de animais na experimentação e na educação, cabe ao Centro Brasileiro de Validação de Métodos Alternativos (BraCVAM), criado pela Agência Nacional de Vigilância Sanitária (Anvisa), no ano de 2013. Vale salientar que são empregados critérios estabelecidos para a validação dos métodos alternativos, em um processo que envolve estágios de desenvolvimento, pré-validação, validação e revisão por especialistas, o que garante a reprodutibilidade e a relevância do teste (Barker-Haliski \& White, 2020; Jeong et al., 2020). Um total de 24 métodos alternativos foram reconhecidos pelo CONCEA (2014) com aceitação regulatória internacional e validação formal por centros internacionais de validação seguindo as guias e protocolos (Test Guideline-TG) de experimentos científicos endossados pela Organization for Economic Cooperation and Development (OECD) (Organização para Cooperação e Desenvolvimento Econômico (OCDE) (CONCEA, 2014). O CONCEA ainda estabeleceu a obrigatoriedade de implementação desses métodos alternativos em um prazo máximo de cinco anos, proibindo a apresentação de resultados de testes com animais quando houverem métodos alternativos reconhecidos (CONCEA, 2014).

Projetos contemporâneos de pesquisa biomédica estão cada vez mais aplicando os princípios dos "3Rs" para a realização de experimentação animal, ou seja, com foco na substituição, redução e refinamento (Madden et al., 2012; Peric et al., 2015).

Um estudo experimental bem sucedido requer o planejamento de fatores como o tamanho da amostra, ou seja, o número de animais, para possibilitar a distinção entre efeitos reais e descobertas casuais. Este cálculo está intrinsecamente relacionado ao grau de confiabilidade da pesquisa, considerando que quanto maior o poder estatístico da pesquisa menor a taxa de resultados falsos negativos e também falsos positivos. Madden et al. (2012) elucidaram os principais fatores que devem ser considerados antes de iniciar o teste in vivo. Segundo esses autores, é necessário determinar a necessidade de realização do teste, selecionar e identificar os compostos, organizar a ordenação hierárquica dos ensaios, escolher o melhor design experimental estatístico, treinar o operador e, por fim, construir relatórios e recomendações. Experimentos bem planejados, que utilizam um número adequado de animais, aliados à uma análise estatística adequada, garantem a robustez e validade dos resultados experimentais. Por outro lado, em experimentação animal, particularmente pesquisas pré-clínicas para o desenvolvimento de drogas e descoberta de mecanismos de doenças, podem não predizer ensaios clínicos bem-sucedidos de agentes terapêuticos devido a fatores como erro aleatório, mecanismos intrínsecos à doença diferentes em humanos e modelos animais usados e, viés e falta de rigor no desenho experimental. Uma pesquisa experimental rigorosa deve envolver conduta e análise que evitem vieses levando-se em consideração os aspectos apresentados na tabela 1.

Ainda no que se refere à experimentação animal, ressalta-se que a identificação e alocação dos animais devem ser feitas por uma pessoa diferente daquela que conduz o experimento (observação e avaliação dos resultados), para garantir o cegamento e evitar preconceitos. Co-alojar machos e fêmeas, ou até mesmo alojar ambos os sexos no mesmo ambiente podem afetar o resultado, principalmente em avaliações comportamentais (Smith et al., 2017).

Sabe-se que pequenas melhorias em projetos experimentais podem levar a economia importantes em recursos e no uso de animais ao longo do tempo. A otimização dos experimentos pode ser influenciada por aspectos do meio ambiente, como alojamento, ocupação, método de contenção, tamanho do grupo, 
material de cama e fatores específicos do experimento, como tempo de doses, métodos ou via de administração e tempo de observação (Madden et al., 2012). Recentemente, Bonapersona et al. (2019), considerando o fato de que novas opções de tratamento desenvolvidas em modelos animais frequentemente falham em ensaios clínicos em humanos e, tendo em vista as questões éticas e de viabilidade, lançaram uma ferramenta de código aberto, baseada na web, o RePAIR (redução de animais por informações de pesquisa prévia), que aumenta o poder estatístico da pesquisa sem aumentar o número de animais, podendo este ser reduzido em até $49 \%$.

Tabela 1. Fatores intrínsecos à experimentação animal bem-sucedida.

\begin{tabular}{ll}
\hline Fator & Questões a serem consideradas \\
\hline $\begin{array}{l}\text { Objeto do estudo vs Desenho } \\
\text { experimental }\end{array}$ & $\begin{array}{l}\text { Como e com que frequência o medicamento é administrado (isso pode altera a escolha da } \\
\text { espécie, por exemplo, i.v. diariamente pode ser problemático em camundongos; oral pode ser } \\
\text { um problema em ruminantes. } \\
\text { Possibilidade de construir uma curva de resposta à dose (isto pode alterar o desenho do estudo, } \\
\text { números de amostra e método de análise de dados) }\end{array}$ \\
\hline $\begin{array}{l}\text { Escolha de } \\
\text { resultado }\end{array}$ & $\begin{array}{l}\text { Padronização dos resultados para melhorar a confiabilidade e reprodutibilidade. } \\
\text { Pode haver variações regionais exigindo a especificação dos locais de amostragem. } \\
\text { Pré-determinar os critérios de inclusão/exclusão para avaliação de resultados, podendo até } \\
\text { remover animais para evitar viés de resultados incompletos. }\end{array}$ \\
\hline Animais & $\begin{array}{l}\text { A escolha dos animais (idade, sexo, espécie) depende do objetivo do estudo. } \\
\text { Os resultados podem ser afetados por diferenças na origem dos animais (por exemplo, estresse }\end{array}$ \\
& $\begin{array}{l}\text { no transporte; animais de diferentes fontes podem ter microbiomas díspares). Se os animais } \\
\text { forem provenientes de locais diferentes, deve ser garantido um período de aclimatação } \\
\text { adequado para evitar resultados diferentes. }\end{array}$ \\
& $\begin{array}{l}\text { Forma de criação de animais (por exemplo, os analgésicos interferem na avaliação da dor; todos os } \\
\text { analgésicos, mesmo os opióides, podem alterar a inflamação e, potencialmente, o curso da doença. }\end{array}$ \\
\hline Grupos de controle & $\begin{array}{l}\text { Selecione de grupos de comparação (por exemplo, considere incluir controle 'positivo/saudável' } \\
\text { sem doença, controle 'negativo/máxima' de doença não tratada, bem como grupos induzidos e } \\
\text { tratados com simulação). } \\
\text { O membro contra-lateral pode não ser apropriado como um controle. }\end{array}$ \\
\hline
\end{tabular}

Fonte: Adaptado de Smith et al. (2017).

Modelos computacionais podem ser projetados para simular resultados gerados em testes toxicológicos in vitro e in vivo. Péry et al. (2010) apresentaram uma metodologia in silico de testes toxicológicos, baseada na modelagem QSAR de limites toxicológicos e simulações de Monte Carlo. O referido modelo é capaz de prever corretamente o desempenho de testeS projetados para analisar os efeitos teratogênicos de dois produtos químicos: di(2-etilhexil)ftalato e indometacina. Os mesmos autores reiteram a grande utilidade de modelos computacionais projetados para simular resultados gerados por testes toxicológicos in vitro e in vivo, uma vez que se constitui uma valiosa ferramenta de apoio à tomada de decisão durante a seleção de produtos químicos candidatos para o projeto de novos produtos.

\section{Legislação na experimentação animal}

No Brasil, a prática de crueldade com os animais é vedada pela Constituição Federal de 1988, em seu artigo 225, parágrafo $1^{\circ}$, inciso VII. Esse dispositivo é regulamentado pela Lei 11.794/2008, conhecida como Lei Arouca, que estabelece procedimentos para o uso científico e didático de animais, com normas legais aplicáveis às atividades de criação, manejo e uso de vertebrados vivos, e a Lei 9.605/2008, que versa sobre os crimes ambientais (BRASIL, 2008; Disner, 2019; Medeiros \& Albuquerque, 2015). Também em 2008 ocorre a criação do Conselho Nacional de Controle a Experimentação Animal (CONCEA), órgão colegiado integrante do Ministério da Ciência, Tecnologia e Inovações e Comunicações (MCTI), que formula normas referentes a utilização de forma humanitária de animais em ensino e pesquisa. Além disso, cabe ao CONCEA o credenciamento e licenciamento das instituições para instalação e manutenção de centros de criação, biotérios e laboratórios de experimentação animal (Disner, 2019).

Inspirada no princípio dos 3Rs, foi criada pelo MCTIC, pela Portaria $n^{\circ} 491$, de 3 de julho de 2012, a Rede Nacional de Métodos Alternativos ao Uso de Animais (RENAMA), que garantiu a infraestrutura 
laboratorial e de recursos humanos especializados necessários para a implantação de métodos alternativos ao uso de animais e validar novos métodos no Brasil (BRASIL, 2012).

A vedação da utilização de animais em atividades de ensino e testes laboratoriais com substâncias para o desenvolvimento de produtos cosméticos, de higiene pessoal e perfumes foi objeto do Projeto de Lei $\mathrm{n}^{\circ} 70$ na Câmara Federal em 2014. A partir daí a proibição dos testes em animais para produtos e ingredientes cosméticos foi promulgada em vários estados brasileiros (Disner, 2019).

Em setembro de 2019 encerrou o prazo estabelecido pelo CONCEA, em setembro de 2019, para a substituição obrigatória de 17 métodos tradicionais utilizados em experimentação animal por métodos alternativos reconhecidos pelo referido Conselho, por meio da Resolução Normativa $n^{\circ} 18$, de 24 de setembro de 2014. Outros sete métodos alternativos (avaliação do potencial de irritação e corrosão ocular, do potencial de irritação e corrosão dérmica, de sensibilização dérmica, resposta alérgica, avaliação da absorção dérmica, de toxicidade (aguda, reprodutiva, genotoxicidade e fototoxicidade), e contaminação pirogênica em produtos injetáveis) reconhecidos através da Resolução Normativa $\mathrm{n}^{\circ} 31$, de 18 de agosto de 2016 devem ser implementadas até 2021 (CONCEA, 2014; Disner, 2019).

\section{Comitê de ética}

A perspectiva ética da ciência do bem-estar animal e o debate público a respeito do uso de animais em pesquisas científicas revelam essencialmente duas posições filosóficas que dominam as discussões sobre o uso de animais: a visão utilitarista, que implica uma ponderação dos danos e benefícios para o bem-estar de humanos e animais, e a visão dos direitos dos animais, que pondera que os interesses de um indivíduo nunca deve ser sacrificados em benefício do outro. Apesar de ser o utilitarismo a abordagem dominante na prática da ética animal na sociedade ocidental, este raramente é aplicado em sua forma "purista". Há em muitos casos, uma abordagem utilitarista pluralista, em razão de uma visão híbrida dos argumentos éticos, que são pesados quanto à sua relevância (Graham \& Prescott, 2015).

Vários fatores foram sugeridos para serem indicadores de bem-estar. Entre as primeiras definições científicas propostas estavam as do Comitê Brambell, baseadas na sugestão de que a ausência de dano determina a presença de bem-estar, conhecidas como as 'cinco liberdades' (Brambell, 1965): liberdade de sede, fome e desnutrição, liberdade de desconforto, liberdade de dor, ferimentos e doenças, liberdade para expressar comportamento normal, liberdade de medo e angústia. Posteriormente, a definição de bem-estar animal passou a reconhecer a presença de estados afetivos positivos e negativos em animais (Tannenbaum, 1991), incluindo a presença de sentimentos positivos (Mench, 1993), sobretudo relacionado na capacidade do animal de lidar com seu ambiente (Broom, 1986), levando em consideração a dinâmica da capacidade adaptativa do indivíduo (Ohl \& Van der Staay, 2012).

Com a crescente demanda na utilização de animais em pesquisas médicas e biomédicas e em instituições de ensino no país foram criados os comitês de ética, com o objetivo de analisar, discutir e aprovar ou não, projetos submetidos para uso de animais em suas pesquisas baseado em princípios nacionais e internacionais de segurança e bioética.

As exigências para limitação do uso indiscriminado de animais para fins científicos ganham ainda mais respaldo quando conselhos e comitês de ética das universidades passam a considerar métodos mais atualizados de experimentação. Conforme já mencionado, o CONCEA reconhece 24 métodos alternativos em experimentação animal, o que representa um grande avanço na discussão ética a esse respeito. No que se refere a pesquisa básica, existe uma tendência de aceitação por parte dos Comitês de Ética de estudos que estejam de acordo com o preceito dos 3Rs (Disner, 2019).

\section{Considerações finais}

Como observado no decorrer deste estudo, são vastos os benefícios alcançados com o uso de animais na experimentação. Entretanto, vale ressaltar que aspectos referentes aos tipos de pesquisa e modelos experimentais tem que ser levados em consideração, respeitando sempre os princípios legais e éticos, o que reflete na validade e aceitabilidade dos resultados. Com a incorporação do princípio dos $3 \mathrm{R}$ nos modelos experimentais oferece uma maior aceitabilidade frente à comunidade científica e população em geral. Cada país é responsável pela criação de suas leis e regulamentos que norteiam o uso de animais em pesquisa, seguindo premissas do que é estabelecido pela comunidade internacional. 


\section{Referências bibliográficas}

Balls, Michael, Combes, R. D., \& Worth, A. P. (2018). Alternative toxicity test methods: lessons learned and yet to be learned. In M Balls, R. D. Combs, \& A. P. Worth (Eds.), The Hhstory of alternative test methods in toxicology (pp. 317-323). Elsevier. https://doi.org/10.1016/B978-0-12-813697-3.00034-2.

Barker-Haliski, M., \& White, H. S. (2020). Validated animal models for antiseizure drug (ASD) discovery: advantages and potential pitfalls in ASD screening. Neuropharmacology, 167, 107750. https://doi.org/10.1016/j.neuropharm.2019.107750.

Bonapersona, V., Hoijtink, H., Sarabdjitsingh, R. A., \& Joëls, M. (2020). RePAIR: a power solution to animal experimentation. BioRxiv, 864652. https://doi.org/10.1101/864652.

Bones, V. C., \& Molento, C. F. M. (2012). Alternativas ao uso de animais de laboratório no Brasil. Veterinária Em Foco, 10(1), 103-112.

Brambell, F. W. R. (1965). Report of the Technical Committee to Enquire into the Welfare of Animals kept under Intensive Husbandry Systems. HM Stationery Office.

BRASIL. Ministério da Ciência, Tecnologia, Inovações e Comunicações. Portaria nº 491, de 3 de julho de 2012. Institui a Rede Nacional de Métodos Alternativos - Renama. Diário Oficial [da] União. 2012 jul. 4.

BRASIL. Lei $\mathrm{n}^{\mathrm{o}}$ 11.794, de 8 de outubro de 2008. Estabelece os procedimentos para o uso científico de animais. Diário Oficial [da] União. 2008 nov. 9. 9.

Broom, D. M. (1986). Indicators of poor welfare. British Veterinary Journal, 142(6), 524-526.

CONCEA. Conselho Nacional de Controle de Experimentação Animal. Resolução normativa $n^{\circ} 18$, de 24 de setembro de 2014, reconhece métodos alternativos ao uso de animais em atividades de pesquisa no Brasil. Diário Oficial, Brasília, DF, 25 set. 2014.

Disner, G. R. (2019). Métodos alternativos à experimentação animal: aspectos éticos, históricos e legais no Brasil. Evidência, 19(2), 259-274. https://doi.org/10.18593/eba.v19i2.20964.

Doke, S. K., \& Dhawale, S. C. (2013). Alternatives to animal testing: a review. Saudi Pharmaceutical Journal, 23, 223-229.

Franco, A. L., Nogueira, M. N. M., Sousa, N. G. K., da Frota, M. F., Fernandes, C. M. S., \& Serra, M. C. (2014). Pesquisas em animais: uma reflexão bioética. Acta Bioethica, 20(2), 247-253.

Garcia Filho, S. P., Martins, L. L., Reis, A. C. G., Pacheco, M. R., \& Machado, M. R. F. (2013). Estrutura, ultraestrutura e morfometria da veia cava de paca (Cuniculus paca Linnaeus, 1766) criada em cativeiro. Pesquisa Veterinária Brasileira, 33, 683-687. https://doi.org/10.1590/S0100-736X2013000500021.

Goldim, J. R., \& Raymundo, M. M. (1997). Pesquisa em saúde e direitos dos animais. Porto Alegre: HCPA.

Graham, M. L., \& Prescott, M. J. (2015). The multifactorial role of the 3Rs in shifting the harm-benefit analysis in animal models of disease. European Journal of Pharmacology, 759, 19-29. https://doi.org/10.1016/j.ejphar.2015.03.040.

Guimarães, M. V., Freire, J. E. C., \& Menezes, L. M. B. (2016). Utilização de animais em pesquisas: breve revisão da legislação no Brasil. Revista Bioética, 24, 217-224. https://doi.org/10.1590/198380422016242121.

Isola, J. G. M. P., Moraes, P. C., Rahal, S. C., \& Machado, M. R. F. (2013). Morfologia, ultraestrutura e morfometria do tegumento da paca (Cuniculus paca Linnaeus, 1766) criada em cativeiro. Pesquisa Veterinária Brasileira, 33, 674-682. https://doi.org/10.1590/S0100-736X2013000500021.

Jeong, Y., Jeon, S., \& Eun, S. (2020). Validation of animal models for facial transplantation research. Transplantation Proceedings, 52(6), 1884-1890. https://doi.org/10.1016/j.transproceed.2020.01.168.

Madden, J. C., Hewitt, M., Przybylak, K., Vandebriel, R. J., Piersma, A. H., \& Cronin, M. T. D. (2012). Strategies for the optimisation of in vivo experiments in accordance with the 3Rs philosophy. Regulatory Toxicology and Pharmacology, 63(1), 140-154. https://doi.org/10.1016/j.yrtph.2012.03.010.

Magalhães, L. E. (2012). A ciência e os animais de laboratório. Revista Da Sociedade Brasileira de Ciência Em Animais de Laboratório, 1(1), 7-13.

Matthiessen, L., Lucaroni, B., \& Sachez, E. (2003). Towards responsible animal research: Addressing the ethical dimension of animal experimentation and implementing the 'Three Rs' principle in biomedical research. EMBO Reports, 4(2), 104-107. https://doi.org/10.1038/sj.embor.embor745. 
Medeiros, F. L. F., \& Albuquerque, L. (2015). Experimentação animal: um combate jurídico nas universidades brasileiras. Revista Internacional Interdisciplinar INTERthesis, 12(1), 65-83. https://doi.org/10.5007/1807-1384.2015v12n1p65.

Mench, J. A. (1993). Assessing animal welfare: An overview. Journal of Agricultural \& Environmental Ethics, 6, 68-75.

Morales, M. M. (2008). Métodos alternativos à utilização de animais em pesquisa científica: mito ou realidade? Ciência e Cultura, 60(2), 33-36.

Ohl, F., \& Van der Staay, F. J. (2012). Animal welfare: At the interface between science and society. The Veterinary Journal, 192(1), 13-19.

Peric, M., Dumic-Cule, I., Grcevic, D., Matijasic, M., Verbanac, D., Paul, R., Grgurevic, L., Trkulja, V., Bagi, C. M., \& Vukicevic, S. (2015). The rational use of animal models in the evaluation of novel bone regenerative therapies. Bone, 70, 73-86. https://doi.org/10.1016/j.bone.2014.07.010.

Péry, A. R. R., Desmots, S., \& Mombelli, E. (2010). Substance-tailored testing strategies in toxicology: an in silico methodology based on QSAR modeling of toxicological thresholds and Monte Carlo simulations of toxicological testing. Regulatory Toxicology and Pharmacology, 56(1), 82-92. https://doi.org/10.1016/j.yrtph.2009.09.009.

Schatzmayr, H. G., \& Müller, C. A. (2008). As interfaces da bioética nas pesquisas com seres humanos e animais com a biossegurança. Ciência Veterinária Tropical, 11(1 Suppl), 130-134.

Smith, M. M., Clarke, E. C., \& Little, C. B. (2017). Considerations for the design and execution of protocols for animal research and treatment to improve reproducibility and standardization:"DEPART wellprepared and ARRIVE safely." Osteoarthritis and Cartilage, 25(3), 354-363. https://doi.org/10.1016/j.joca.2016.10.016.

Tannenbaum, J. (1991). Ethics and animal welfare: The inextricable connection. Journal of the American Veterinary Medical Association, 198(8), 1360-1376.

Tréz, T. A. E. (2010). Refining animal experiments: the first Brazilian regulation on animal experimentation. Alternatives to Laboratory Animals, 38(3), 239-244. https://doi.org/10.1177/026119291003800308.

Tréz, T. A. E., \& Rosa, V. V. L. (2013). Uma abordagem Fleckiana da experimentação animal na educação científica superior. Alexandria: Revista de Educação Em Ciência e Tecnologia, 6(3), 27-60.

Yellowlees, A., \& Perry, R. H. J. (2015). Estimating vaccine efficacy using animal efficacy data. European Journal of Pharmacology, 759, 63-68. https://doi.org/10.1016/j.ejphar.2015.03.034.

Histórico do artigo:

Recebido: 8 de março de 2021 Aprovado: 23 de abril de 2021
Licenciamento: Este artigo é publicado na modalidade Acesso Aberto sob a licença Creative Commons Atribuição 4.0 (CC-BY 4.0), a qual permite uso irrestrito, distribuição, reprodução em qualquer meio, desde que $\mathrm{o}$ autor e a fonte sejam devidamente creditados. 